\title{
Translational precision medicine: an industry perspective
}

\author{
Dominik Hartl ${ }^{1,2^{*}}$, Valeria de Luca', Anna Kostikova' , Jason Laramie ${ }^{3}$, Scott Kennedy ${ }^{3}$, Enrico Ferrero ${ }^{1}$, \\ Richard Siegel ${ }^{1}$, Martin Fink ${ }^{1}$, Sohail Ahmed ${ }^{4}$, John Millholland ${ }^{5}$, Alexander Schuhmacher ${ }^{6}$, Markus Hinder $^{1}$, \\ Luca Piali ${ }^{7}$ and Adrian Roth ${ }^{7}$
}

\begin{abstract}
In the era of precision medicine, digital technologies and artificial intelligence, drug discovery and development face unprecedented opportunities for product and business model innovation, fundamentally changing the traditional approach of how drugs are discovered, developed and marketed. Critical to this transformation is the adoption of new technologies in the drug development process, catalyzing the transition from serendipity-driven to data-driven medicine. This paradigm shift comes with a need for both translation and precision, leading to a modern Translational Precision Medicine approach to drug discovery and development. Key components of Translational Precision Medicine are multi-omics profiling, digital biomarkers, model-based data integration, artificial intelligence, biomarker-guided trial designs and patient-centric companion diagnostics. In this review, we summarize and critically discuss the potential and challenges of Translational Precision Medicine from a cross-industry perspective.
\end{abstract}

Keywords: Translational medicine, Precision medicine, Drug development, Biomarkers, Multi-omics, Modeling, Artificial intelligence, Pharmaceutical industry, Digital biomarkers, Companion diagnostics

\section{Background}

Traditionally, drug development in large pharmaceutical companies is regarded as a conservative and riskaverse discipline with highly regulated processes and slow adaptation to external innovation. However, in a rapidly evolving healthcare ecosystem, new technologies and innovative concepts of how to leverage them are needed to accelerate clinical trials, lower attrition rates, mitigate research and development (R\&D)-related risks and overall improve pharmaceutical $R \& D$ productivity $[1,2]$. Critical for future $R \& D$ success is the combination of transformative therapeutic concepts and drug targets with first-in-class potential, tailored digital technologies and patient-centric drug development, linked

\footnotetext{
*Correspondence: dominik.hartl@novartis.com; dominik.hartl@med.unituebingen.de

${ }^{1}$ Novartis Institutes for BioMedical Research, Basel, Switzerland

Full list of author information is available at the end of the article
}

to a broader paradigm shift from one-size-fits-all medicine towards precision medicine (the right medicine, for the right patient, at the right dose, at the right time) $[3,4]$. While precision medicine is an appealing concept, there are several core challenges for implementation from bench to the bedside, as discussed previously [5-7].

One of the major bottlenecks for drug development is translation [8], particularly at the interface of drug discovery and early clinical development, referred to as the Translational Gap [8-10]. To close this gap and foster translational science, the National Institutes of Health (NIH) has established the National Center for Advancing Translational Sciences, a core hub to drive and integrate innovative translational activities across academia, industry and non-profit organizations [11]. Translational medicine as defined by the European Society for Translational Medicine [12] integrates several R\&D tools to bridge the translational gap and guide early drug development. Since translational medicine

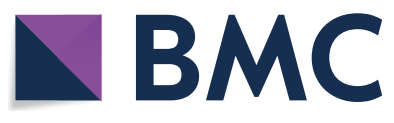

c) The Author(s) 2021. This article is licensed under a Creative Commons Attribution 4.0 International License, which permits use, sharing, adaptation, distribution and reproduction in any medium or format, as long as you give appropriate credit to the original author(s) and the source, provide a link to the Creative Commons licence, and indicate if changes were made. The images or other third party material in this article are included in the article's Creative Commons licence, unless indicated otherwise in a credit line to the material. If material is not included in the article's Creative Commons licence and your intended use is not permitted by statutory regulation or exceeds the permitted use, you will need to obtain permission directly from the copyright holder. To view a copy of this licence, visit http://creativeco mmons.org/licenses/by/4.0/. The Creative Commons Public Domain Dedication waiver (http://creativecommons.org/publicdomain/ zero/1.0/) applies to the data made available in this article, unless otherwise stated in a credit line to the data. 
and precision medicine approaches in drug development are overlapping and intertwined, we use here the term Translational Precision Medicine to refer to this emerging discipline.

The Translational Precision Medicine concept integrates core components from both translational medicine (mechanism-based early drug development) and precision medicine (patient-centric late drug development) into an end-to-end biomarker-guided drug development cycle. Critical success factors for Translational Precision Medicine are (i) the translation of mechanisms from research to early clinical development (forward translation/bench-to-bedside), (ii) the back-translation from late clinical development insights to drug discovery (reverse translation/backtranslation/bedside-to-bench) [13], (iii) data-driven mechanism-indication pairing [14], (iv) the translation of omics signatures into clinically-relevant biomarkers and endotypes [15] and (v) the development of patient-tailored companion diagnostics and precision medicines [3]. Here we focus on the following key components of Translational Precision Medicine (Fig. 1):

- Multi-omics profiling

- Biomarker-guided trial designs

- Model-based data integration

- Artificial Intelligence (AI)

- Digital biomarkers

- Patient engagement.

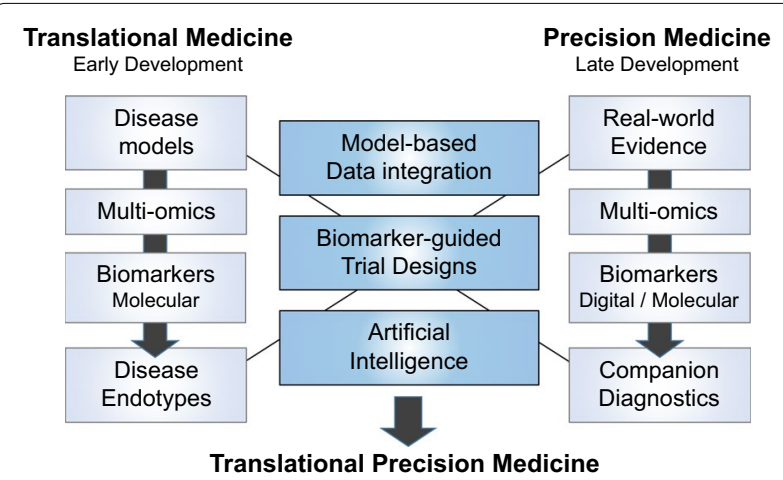

Fig. 1 Interface position of Translational Precision Medicine in bridging translational medicine (early development) and precision medicine (late development). Disease models, multi-omics and molecular biomarkers are used to define disease endotypes. Real-world evidence, multi-omics, biomarkers (digital and molecular) and companion diagnostics are instrumental for the implementation of precision medicine. Model-based data integration, biomarker-guided trial designs and artificial intelligence are key data-driven tools for the integration of mechanism-centric translational medicine and patient-centric precision medicine

\section{Multi-omics profiling}

Clinical data can be classified as phenotypic (such as demographics, physiologic assessments, disease scorings, imaging, health questionnaires, digital patient assessments) or molecular (such as genomics, transcriptomics, proteomics, metabolomics). Capturing comprehensive phenotypic data associated with a certain disease can be referred to as phenotyping (or phenomics), which is the traditional and most common approach to classify diseases irrespective of the biological origins of disease. Utilizing datasets to define disease subtypes at the molecular level can be referred to as endotyping, as exemplified in respiratory medicine $[16,17]$ or oncology [18]. The National Academy of Sciences of the USA campaigned for a new, molecularly-informed taxonomy to define diseases based on molecular endotypes rather than traditional clinical symptoms [19]. However, endotyping requires deep pathophysiological disease insights and large molecular datasets to be successful. Within the last two decades, high-throughput omics technologies have provided the basis for endotyping and data-driven medicine [20,21]. With the rapid advances of sequencing technology, genetics has revolutionized our understanding of monogenic diseases, such as cystic fibrosis [22] or mutation-driven cancers [23], but most human diseases are polygenic and consequently more complex to dissect. To approach these diseases at the genetic level, polygenic risk scores hold promise to predict genetic predisposition to disease or therapeutics [24], particularly if combined with electronic health records (EHRs) [25]. Translational genomics aims to combine genetic and clinical data as a foundation for precision medicine approaches [7]. Besides genetics, particularly proteomics $[26,27]$ are gaining momentum for clinical biomarkers and drug development [28]. Unlike mass spectrometry, next-generation proteomic detection principles, such as aptamer-based technologies [29], typically require lower amounts of material (down to $1 \mathrm{ul} /$ sample) and can be more readily applied to large patient cohorts to identify causal proteins as candidates for therapeutic targeting [28, 30-33]. Compared to more established omics layers, such as transcriptomics, proteomics offer the benefit of measuring protein levels directly, thus facilitating the translation to the clinic where protein biomarkers are most commonly used.

Beyond single omics technologies, multi-omics profiling platforms are emerging, including genomics, epigenomics, transcriptomics, proteomics, lipidomics, metabolomics, microbiomics and others [34, 35]. Multi-omics profiling integrates several biological layers, allowing researchers to fully appreciate the interplay between genetics, gene regulation and proteins, and to obtain a more complete picture of the molecular patterns 
underpinning complex diseases. Thus, multi-omics are well positioned to enable the identification of key disease nodes where multiple layers converge, maximizing the chances to identify novel drug targets, endotypes or biomarkers. Networks offer an effective way to integrate and visualize the output of multi-omics analyses, particularly when the evidence does not converge at the level of a single gene, but within a biological pathway [36], and network propagation approaches can be applied to leverage network topology for the identification of key nodes [37]. Multi-omics are further essential for $N$-of- 1 trials [38], for understanding drug-drug interactions and for the design of therapeutic drug combinations [39-41]. Despite this potential, there are several caveats and limitations of multi-omics when applied to clinical drug development:

- Omics technologies assess large numbers of genes/ proteins, often in a semi-quantitative manner, and are highly sensitive to pre-analytical processes [42] such as batch effects [43]. Consequently, to build robust cases for clinical adoption, it is essential to include appropriate controls in the experimental design and to validate top hits by orthogonal quantitative methods $[43,44]$.

- Merging different multi-omics datasets [45] into a single data repository poses challenges to data transfer, integration and harmonization given different data formats and data fragmentation. Moreover, analyzing large complex datasets, such as single-cell multi-omics [46], increases the chance for false positives and necessitates appropriate data processing, normalization and analysis with appropriate statistical methods [44, 47].

- Clinical trial feasibility $[15,48]$, especially for multicenter and tissue-derived omics, remains a challenge. Restricting the number of well-selected clinical sites, strict standard operating procedures (SOPs), crosssite controls and qualified analytical core facilities are essential for robust data generation. Well-curated biobanks [49] are further pivotal to link multi-omics data to disease characteristics and clinical trial outcomes. Alignment on human biosample accessibility, FAIR data principles [50] and dissemination policies are also key for successful multi-omics collaboration networks.

\section{Biomarker-guided trial designs}

Biomarkers are defined by the Biomarkers Definitions Working Group of the NIH/FDA, as "a characteristic that is objectively measured and evaluated as an indicator of normal biological processes, pathogenic processes, or pharmacologic responses to a therapeutic intervention" [51]. In drug development, biomarkers are broadly used to inform on target engagement, pathway activation, pharmacokinetic/pharmacodynamic (PK/PD) modeling and dosing rationales, diagnosis/patient selection, disease stratification, prognosis and prediction as well as monitoring disease, safety and treatment efficacy. Biomarkers are classified into molecular, cellular, physiological, imaging and digital modalities. As clinical trial endpoints, biomarkers provide the advantage of being quantitative and objective measures of (patho)biology in contrast to physician-based assessments which tend to be subjective and variable. Biomarkers are key to translate PD responses across species and to bridge the translational gap in early drug development $[9,10,52]$, particularly for multifactorial systemic diseases [53] such as systemic immune-mediated diseases. From a drug development perspective, the longitudinal analysis of the AstraZeneca small molecule portfolio (five-dimensional (5R) framework) demonstrated that the inclusion of biomarkers into early drug development ( $\mathrm{Ph} 2$ studies) was associated with active or successful projects in contrast to comparable projects without biomarkers [2].

The development of a new biomarker is a complex, multistep and iterative process, including biomarker discovery (often based on omics data), pre-analytical validation, assessing different biofluids (best proximal to the disease), analytical validation and finally clinical validation and utility [48]. For each new drug target and disease indication, several biomarker modalities and candidates are usually explored to narrow-down on the drug targetand indication-relevant ones, as discussed here for autoimmune diseases such as rheumatoid arthritis [54]. For biomarker use in clinical trials it is critical to define the context-of-use (CoU) $[15,55,56]$. CoU range from diagnostic, safety monitoring, PD response, to predictive and prognostic biomarker applications. For a detailed list of biomarker $\mathrm{CoU}$, the reader is referred to the FDA-NIH biomarker working group and its related online resource BEST (Biomarkers, Endpoints and other Tools) [51]. Prognostic and predictive $\mathrm{CoU}$ are essential for clinical drug development: prognostic biomarkers at baseline are indicative of disease outcome independent from interventions (important to identify patients on high-risk for trial enrichment), whereas predictive biomarkers at baseline are indicative of response to a specific treatment (response prediction).

There are two basic paths how to integrate biomarkers in drug development: (1) within the context of a specific drug development program or (2) the official FDA biomarker qualification program (BQP). The specific drug development program path is the most common strategy pursued in pharmaceutical industry, 
where the drug developer / sponsor includes selected biomarkers in clinical trials, mainly for internal decision-making and is responsible for all aspects of the biomarker development. The FDA BQP is required to qualify biomarkers as general drug development tools [57] to make them applicable for multiple drug development programs and to qualify them as regulatory drug approval tools, which is a formal and lengthy process usually involving consortia composed of multiple academic and industry partners. In the FDA BQP, the candidate biomarker is qualified for a pre-specified CoU. The FDA provides an updated online list of BQP-qualified biomarkers [58]. Of note, qualifying a biomarker for a $\mathrm{CoU}$ via the BQP or qualifying a specific test measuring a biomarker are two different and independent approaches. For biomarker test/assay qualification, e.g. to develop a companion diagnostic assay (see below), pre-analytical and assay performance characteristics are key. Beyond the US/FDA, other regional/national biomarker guidances and regulatory frameworks, such as guidances from European Medicines Agency (EMA), Asian-Pacific (APAC) regulators, National Medical Products Administration (NMPA) and/or Pharmaceuticals and Medical Devices Agency (PDMA), have to be taken into account for biomarker qualification and clinical implementation.

In general, the level of impact that biomarkers can have depends on three key factors: (i) the validation and qualification status of the biomarker, (ii) the $\mathrm{CoU}$ and (iii) the scientific evidence linking the biomarker with the CoU. Biomarkers in clinical trials are mostly used as exploratory endpoints to explore new mechanistic hypothesis and inform internal decision making. If biomarkers are deemed more impactful and clinically relevant, biomarkers are used as secondary or primary clinical endpoints. Typical examples here are physiological biomarkers like blood pressure, clinically-established protein biomarkers such as C-reactive protein, or imaging readouts. If there is convincing evidence from independent epidemiological studies and clinical trials that biomarkers correlate closely with clinical outcome assessments, biomarkers can be considered to substitute for a clinical endpoint as surrogate endpoints, which have a major relevance for diseases with outcomes that take a long time to capture using traditional clinical endpoints. Examples here are systolic blood pressure for occurrence of stroke or lowdensity lipoprotein cholesterol levels for occurrence of heart attacks. For implementation of biomarkers in pharmaceutical industry trials, several drug development aspects have to be further taken into account, including informed consent/data protection considerations, clinical trial logistics/feasibility, impact on clinical decision-making and cost-effectiveness [48, 59-61].
To actively guide clinical trial flows, biomarker-guided trial designs are the method of choice [62,63], which are particularly useful for novel clinical trial designs using master protocols (basket, umbrella and adaptive platform trials) [64]. For a comprehensive overview on biomarkerguided trial designs, the reader is referred to the $B i G T e D$ online resource [65]. For biomarker-guided trial designs, biomarkers should be analyzed in Clinical Laboratory Improvement Amendments (CLIA) certified (for US) or equivalent (non-US) labs. The two most commonly applied biomarker-guided trial designs are stratification [66] and enrichment [67]. Biomarker-based stratification, or stratified randomization, means that biomarkers are measured in all patients prior to randomization and are used to proportionally/equally balance treatment vs placebo arms with respect to biomarker status. Biomarkerstratified designs have the advantage that patients are not excluded if they are biomarker-negative. The next more stringent level of biomarker trial design is enrichment. For that design, inclusion of the individual patient into the clinical trial is depending on a defined biomarker assessment. Quality requirements for biomarkers and analytical labs are higher when using this approach, as protocol-defined treatment decisions depend directly on the biomarker. Enrichment designs can be especially useful for situations when it is not ethically justified to treat biomarker-negative patients based on biomarkerresponse and/or biomarker-safety relationships, such as CYP metabolism. The recent FDA guidance on enrichment [68] should be taken into account that recommends smart enrichment, adaptive enrichment and the inclusion of a biomarker-negative population in at least one trial before NDA/MAA submission (with defined exceptions). Besides stratification and enrichment designs, other more complex biomarker-guided trials designs are summarized as biomarker-strategy designs [65]. All biomarker-guided trial designs can be implemented in non-adaptive or adaptive settings. The latter provides more flexibility for the trial, yet is also more challenging to implement. Apart from interventional biomarkerguided trial designs, non-interventional (observational) biomarker-guided trial designs using master protocols have been proposed recently in the oncology field (Master observational trials) [69].

Biomarker-guided trial designs ultimately pave the way towards precision medicine, i.e. tailoring drug development to specific patient characteristics $[3,70]$. In 2015, the US government launched a Precision Medicine Initiative [71, 72]. Precision medicine focuses on individual rather than average responses to therapy and led to the concept of $\mathrm{N}$-of- 1 trials [38], ideally based on longitudinal multi-omics data. While precision medicine approaches are already widely implemented in 
oncology and rare genetic diseases, other therapeutic areas have just begun to tailor drug development based on these principles [3]. Biomarkers can enable precision medicine through the development of companion diagnostics [73-75], mainly established in oncology [76]. Companion diagnostics are classified as in vitro diagnostic (IVD) medical devices (IVD class I, II or III) and are typically co-developed with the drug to increase response rates by lowering the numbers-needed-totreat and/or to spare patients exposure to drugs that have a high probability to fail or even cause harm. The development path for an exploratory biomarker to a full IVD companion diagnostic is complex, requires at-risk investments and should start early in drug development in close alignment with health authorities [74, 75, 77, 78]. Companion diagnostics should be broadly available and accessible to relevant healthcare professionals for clinical routine use. A list of cleared or approved companion diagnostic devices is provided by FDA [79]. Companion diagnostics [80] are strictly regulated by health authorities/FDA [81] and are differentiated from complementary diagnostics $[82,83]$ as they are essential for treatment decisions, whereas complementary diagnostics just support treatment decisions. As biopsy-derived tissue is often challenging to obtain from non-oncology patients, "liquid biopsies" (derived from peripheral blood/serum/plasma) are a major domain of companion diagnostics, yet assay performance characteristics, such as sensitivity and specificity, are key for success in that area. Figure 2 illustrates the flow from multi-omics-based endotyping, over biomarker-guided trial designs to companion diagnosticsbased precision medicine approaches.

To increase the benefit-risk ratio of drug candidates, safety aspects are increasingly becoming an integral part of biomarker-guided precision medicine approaches. For example, the observation that patients receiving checkpoint inhibitor therapy experiencing immune-related adverse events also exhibit an improved treatment response was recently shown to be related to a polygenic risk score [84]. Furthermore, for the first time, a polygenic risk score could be established for the prediction of

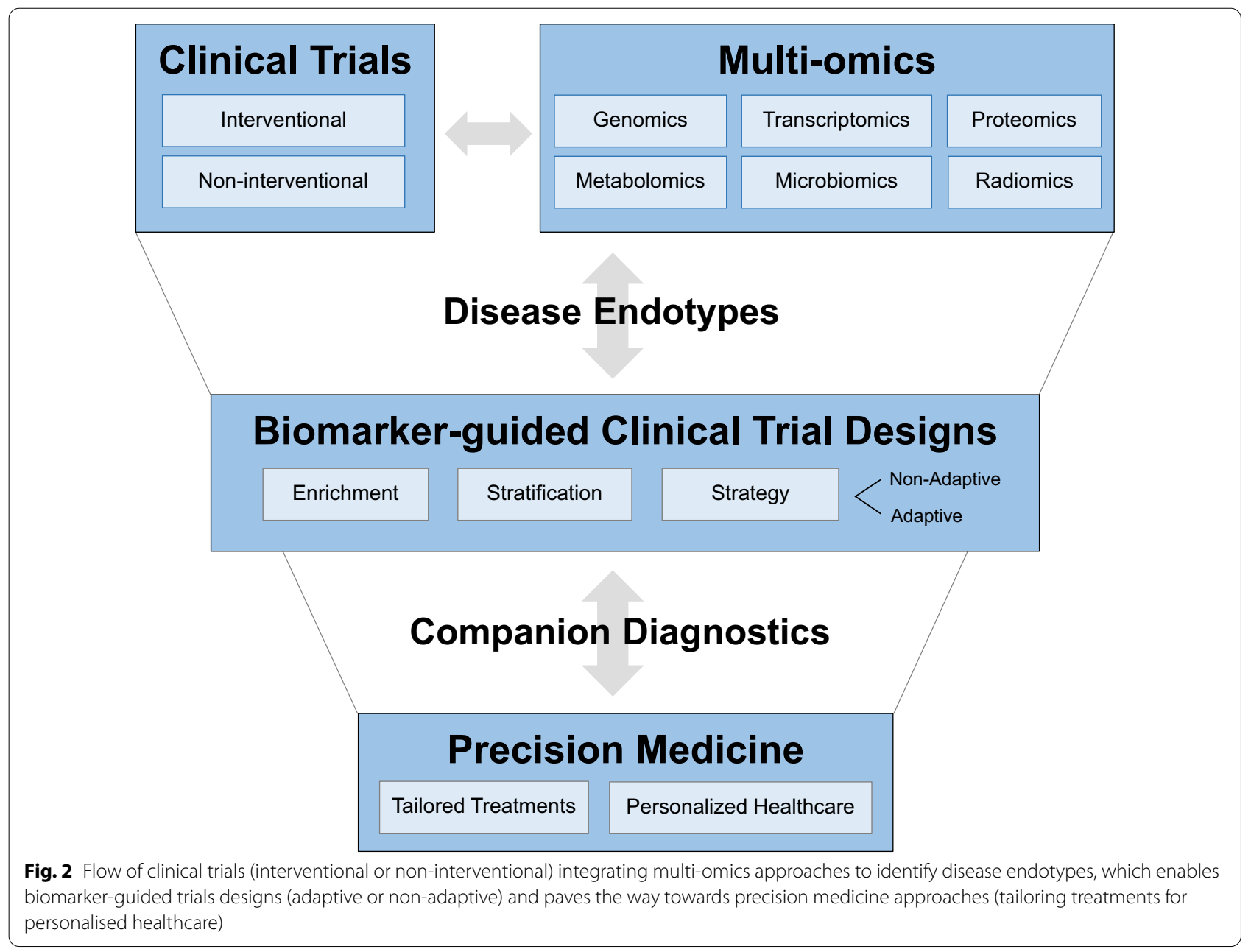


drug-induced liver injury, a common and very difficult to predict adverse event in the clinic [85].

When viewed in combination, biomarker-guided trial designs provide ideal tools to catalyze the transition from an empirical and physician-centric to a data-driven and patient-centric precision medicine approach [70, 86, 87]. However, technical (companion diagnostic assay development), clinical (complex biomarker-guided trial designs, master protocols) and regulatory (requirements for companion diagnostics as medical devices) hurdles have to be tackled, particularly in non-oncology indications [83, 88].

\section{Model-based data integration}

Given the small size and low number of samples per subject in pre-clinical experiments as well as early clinical trials, analyzing and leveraging biomarker data in translational medicine remains challenging. One way for improving the statistical power for detecting signals is to use longitudinal (i.e. time-dependent) model-based data integration. Mathematical models are used to describe the time course of $\mathrm{PK}$ and $\mathrm{PD} /$ biomarker results for better understanding of the pharmacology and to predict future experimental outcomes [89]. Already in the 1930s, mathematical equations were used to describe PK data [90], but the start of more extensive model-based approaches took off with the development of computers and was brought into drug-development in the 1970s80s. The need has been highest for compounds with a small therapeutic window where dose-individualization was needed. This was especially challenging for compounds with a substantial delay between the exposure and the biomarker PD response. Therefore, the initial approaches for describing inter-subject variability (population "PopPKPD" models) were developed in anesthesiology [91] and for anticoagulants (e.g. warfarin [92]).

Population models most often use simplified model structures to model the observed data (i.e. mainly measured in plasma/blood or ex-vivo). When considering chemotherapies, like methotrexate, questions arose about the tumor-relevant tissue distribution of the compound [93]. Thus, a second class of models, i.e., physiologically-based, were developed to describe the whole Liberation, Absorption, Distribution, Metabolism, Excretion (LADME) processes in more detail for small molecules, where these processes are heavily dependent on their physico-chemical properties [94]. This approach is used to predict drug-drug interactions, but also for scaling from animals to humans in the translational medicine realm - alternatively to PopPK models.

Scaling PK parameters across species is mainly based on allometric scaling, which describes the weightdependence of physiological aspects (volumes, metabolic rates, clearance, etc.) within and between species using power functions with fixed exponents. This works well for PK parameters [93], yet can be challenging for biomarkers due to inter-species differences of pathway expression, production rates or whole physiological networks. These approaches have gained more traction by integrating systems biology and quantitative systems pharmacology [95]. A third concept is to apply physical and biological assumptions, such as monotonous exposure-response, continuity/smoothness of underlying signals or allometric scaling. These models can be used for high-dimensional data (like multi-omics) to reduce noise when multiple sampling time points are available per individual.

Finally, the FDA has established a new framework for model-informed drug development [96]: "FDA is conducting a Model-Informed Drug Development (MIDD) Pilot Program to facilitate the development and application of exposure-based, biological, and statistical models derived from preclinical and clinical data sources, referred to as MIDD approaches. MIDD approaches use a variety of quantitative methods to help balance the risks and benefits of drug products in development. When successfully applied, MIDD approaches can improve clinical trial efficiency, increase the probability of regulatory success, and optimize drug dosing/therapeutic individualization in the absence of dedicated trials." This MIDD pilot program is based on joint discussions between the pharmaceutical industry and the European health authorities/EMA, which led to a paper in 2016 on good modeling practices [97]. The latter includes the concept of the learning-and-confirming circle of modeling as well as drug development, where model-based predictions inform the next study design, e.g., predictions of PK and efficacy or safety biomarkers from animal data into firstin-man studies. The acceptance or even push from health authorities for MIDD approaches [98] indicates the high value of model-based data integration.

\section{Artificial Intelligence}

The amount of data generated and collected in pharmaceutical $R \& D$ is increasing at an unprecedented pace. Combined with improvements in information processing and more powerful hardware, machine learning, deep learning and $\mathrm{AI}$ in general are positioned to disrupt drug discovery and development towards an algorithm-based R\&D [99]. Deep learning has already revolutionized several industries, particularly in the area of image analysis and recognition, while its impact in biomedical $R \& D$ remains to be fully embraced [100]. High-dimensional multi-omics datasets derived from large longitudinal clinical studies provide an ideal ground for the application of machine learning [101] and AI [99]. Examples with an impact on drug discovery and development 
include: target identification [102-104], biomarker discovery [105, 106] and patient endotyping [107, 108]. Machine learning and deep learning algorithms are also powerful analytical methods when applied to digital biomarkers data, allowing to transform longitudinal, multimodal and complex raw data from sensors and connected digital devices into endpoints and clinically-relevant measures $[109,110]$.

Given the rigidity of traditional serendipity- and forward translation-based drug development frameworks, shifting to a new mindset embracing the use of AI for the discovery and development of drugs is a critical success factor for Translational Precision Medicine [111]. A comprehensive cross-industry analysis recently mapped out AI-related activities across major pharmaceutical companies [112], coming to the conclusion that, compared with leading technology companies (e.g., Microsoft, Google), most pharmaceutical organizations are still in an early mature phase of using AI in R\&D. However, an increasing number of healthcare companies have now started their digital journey, building up AI competencies and data literacy across many areas of R\&D [112]. For example, Johnson \& Johnson and Novartis have started to commercialize AI-based products and services in healthcare. Medical AI application focused so far mainly on the diagnosis of disease conditions based on EHRs, digital pathology and biomarkers [113, 114]. To go beyond and fully leverage AI technologies for clinical drug development, it is essential to optimize and validate AI algorithms for use in clinical trials and outcome prediction. AI-powered approaches have the potential to enable precision medicine, particularly in chronic disease conditions, by dissecting complex high-dimensional patient datasets and tailoring drug development [115]. While traditionally regulatory authorities might not have been perceived as enthusiastic about advanced AI models in biomedical $R \& D$, the landscape is evolving rapidly, exemplified by recent developments in the AI-based medical device space $[116,117]$ and the recent FDA pilot program Innovative Science and Technology Approaches for New Drugs (ISTAND) that incentivizes the use of AI-based algorithms to evaluate patients, develop novel endpoints, or inform study designs. Moving forward, it will be critical that pharmaceutical organizations continue to constructively engage early on with regulatory authorities on innovative ways to design and assess clinical trials, including a more widespread use of AI technologies in drug development.

Overall, the impact of AI in drug discovery and clinical development will largely depend on the underlying data, and its intrinsic limitations. AI-based analysis of both multi-omics as well as EHRs depends critically on the quality and quantity of the provided molecular and clinical datasets, key limitations and challenges that need to be overcome in the future.

The near future will show whether and how these emerging AI algorithms will help scientists to (i) identify novel targets or new indications for existing drugs, (ii) uncover latent factors that can inform on disease pathogenesis or drug response, (iii) discover predictive biomarkers enabling patient stratification strategies that can optimize clinical trial designs, and (iv) ultimately impact the drug development value chain. For more detailed overviews of AI in drug discovery and development, we refer the reader to dedicated reviews in this field $[87,101$, $111,118]$.

\section{Digital biomarkers}

The recent evolution of sensor technologies and the widespread use of smartphones and other connected digital products are enabling the comprehensive collection and analysis of health-related data [119-121]. Progress in algorithms and analytical methodologies to transform sensor data into clinical insights have facilitated the rapid development of digital biomarkers [122, 123]. Digital biomarkers are defined as physiological and behavioral measures collected via digital devices (such as portables, wearables, implantables and digestables) that characterize, influence or predict health-related outcomes [124, 125]. Digital biomarkers offer several potential advantages compared to traditional clinical assessments. Objective data can be collected in real-life settings, in a quantitative and unbiased way and on a frequent or continuous basis, resulting in increased statistical power, and enhanced sensitivity and specificity $[122,126]$. In clinical trials, these characteristics allow for lower sample size, fewer study visits, shorter study duration and real-time feedback for early decision-making [120, 122, 126, 127]. Longitudinal digital patient data can be leveraged to advance precision/personalized medicine approaches. Furthermore, the use of digital biomarkers in drug development enables patient centricity, integration of real-world evidence, reduced patient burden of trial participation, increased inclusivity in patient enrollment [121], decentralized trials [128] and better product differentiation [129]. Despite being a promising new technology, a major requirement and challenge for digital biomarkers is to ensure protection of relevant sensitive patient data in the whole process.

Successful examples of digital biomarkers are in the field of neurodegenerative diseases, where traditional clinical outcome measures are sparse, highly variable and rater-dependent [130]. Smartphone-based measurements have been developed and deployed in clinical trials to monitor signs of Parkinson's disease [131, 132]; while features from inertial measurement unit 
features have been recently benchmarked to predict Parkinson's disease severity [133]. Susceptibility/risk biomarkers from computerized cognitive testing are in use to classify adults at high risk of late-onset of Alzheimer's disease [134, 135]. Clinically relevant gait parameters from inertial wearable sensor were identified to assess gait impairment in Huntington disease [136].

While the number of studies involving digital technologies is growing and extending to more technologies, biomarker categories and therapeutic areas [119, 121], the use of digital biomarkers as clinical endpoints is today still in an early research phase due to several layers of complexity. Digital biomarker products are usually the result of the combination of multiple individual hardware (sensors) and software (operating systems and algorithms) components [123]. Hence is it vital to thoroughly verify technology and analytical solutions and clinically validate digital biomarkers in the desired cohorts and context of use, prior to their adoption as clinical endpoints [123, 127, 137]. The majority of current efforts still have an engineering focus and address algorithm development and sensor performance [120]. So far, very limited solutions are undergoing clinical validation.

Transforming digital device data into validated clinical endpoints is a lengthy process, which involves the collaboration of multiple disciplines, from engineering, machine learning, data science, clinical research and regulatory interactions. An open validation framework based on transparency, metadata standards, external validation and data sharing is necessary to harmonize approaches and evaluate and improve digital biomarkers in clinical settings [123]. Recently, multiple concrete efforts have emerged and are shaping and accelerating the development of validated digital biomarkers: (i) guidelines from the Clinical Trials Transformation Initiative (CTTI) [138], the Digital Medicine Society [139] and the EMA [140]; (ii) pragmatic fit-for-purpose validation frameworks [137, 141]; (iii) open-source platforms such as the Digital Biomarker Discovery Pipeline [142]; (iv) open benchmarking challenges [143]; (v) and several Innovative Medicine Initiative (IMI) programs, such as MOBILISE-D [144], IDEA-FAST [145] and RADAR-AD [146].

Future opportunities for digital biomarkers towards patient-centric precision medicine are (i) algorithms based on longitudinal/real-time composite biomarkers from multiple connected technologies and contextual information in real-world settings [123], (ii) integration of molecular/multi-omics and digital biomarkers, and (iii) digital phenotyping for patient stratification [147-149].

\section{Patient engagement}

Since the AIDS pandemic in the 1980s, the way industry interacts with patients has changed fundamentally, from passive recipients to active contributors along the whole drug development value chain. This has been particularly evident in the last decade, where most pharmaceutical companies have started patient engagement groups to actively listen to the patient voice $[150,151]$. Industry has finally realized that patient engagement is not an additional burden, but can improve and actually accelerate drug development. Similarly, health authorities increasingly incorporate the patient voice into their regulatory guidance [152]. For example, The FDA's PatientFocused Drug Development initiative led to the guidance for industry on how to best identify what is important to patients. In Europe, the EMA formed its Patients' and Consumers' Working Party. Engagement with patients, their caregivers, patient experts and patient advocacy groups have been shown to yield benefit for both patients and the industry $[153,154]$. Increased patient involvement in the process ensures that industry focuses on the real medical needs, that study protocols are patientcentric and that new treatments become available faster. Conversely, industry benefits from a more robust identification of patients ' needs, faster conduct of clinical trials, a quicker path to market and overall higher credibility and sustainability [155]. Patients not only have increased their involvement with industry, but likewise with regulatory authorities and sit in governing bodies. Major milestones are the foundation of the International Alliance of Patient's Organizations, the Patient-Centered Outcomes Research Institute [156] and the Patient-focused Medicines Development [157] among several other patient-centric initiatives. In fact, the impact of patient engagement throughout the healthcare ecosystem is driving change at various levels: becoming a credible source for patients themselves, improving access and care, driving $R \& D$ and advocating for policy changes in collaboration with governments.

Traditionally, patient engagement has been mainly considered once a new drug is already on the market. The majority of decisions about the molecule and its clinical development path, including unmet medical needs, have then already been taken by the company. Studies, however, demonstrated that the early integration of the patient perspective, particularly in preclinical research and early development, has the biggest impact on value creation for patients, business and society [158]. As preclinical research is a discipline that usually does not collaborate directly with patients, a change of mindset to include the patient voice already at this stage can be challenging, yet represents the clear future towards patient-led research [159]. A recent paper identified 
key challenges of implementing patient engagement in preclinical research and provided possible solutions to overcome current barriers [160]. In interviews with patient groups, industry and academia conducted by the CTTI, patient representatives identified engagement with research partners as having particularly great benefit. Patient-led organizations are keen to learn more about their diseases and are highly interested to collaborate in research projects and willing to provide their data (anonymized and under strict data protection policies) for research and clinical development [161]. A key recommendation for industry is to engage the patient voice as early as possible from the beginning of the R\&D program to improve trial design and clinical execution [162].

Personalised healthcare (PHC), precision medicine and stratified medicine have been used interchangeably to describe the concept of tailoring treatment to patients based on their individual pathology. With the rise of new diagnostic and data-driven approaches that deepen our understanding of the molecular basis of disease, this centuries-old dream has come closer to reality. Nowadays, the awareness of the potential of PHC is also emerging in the patient community and its meaning goes far beyond precision medicine. PHC comprises everything that allows to tailor treatment and medical care by combining conventional clinical datasets, molecular signatures (such as genetics), environment, lifestyle and personal needs. Some of the key innovations in that area include digital healthcare solutions with technologies connecting digital patient information/EHRs with wearable devices, mobile Apps, telehealth and digital assistants using AI [163], see also the respective chapters above. A major requirement and challenge for that field is to protect relevant sensitive patient data and patient rights in that whole process. Patients, caregivers and healthcare providers are acknowledging the utility and advancement offered by these approaches in key domains, such as patient education, accurate diagnosis, patient outcomes, quality of life, disease prevention and health care value [164], more recently underscored by different initiatives, such as the EU Health Data Space race [165] or the US Precision Medicine initiative [166]. The overall goal in all of this is to make healthcare decisions jointly together with the patient as an integrated $R \& D$ partner.

\section{Conclusions}

Translational Precision Medicine comes with a paradigm shift from a one-size-fits-all to a biomarker-guided patient-centric medicine. Key success factors for adoption of this principle in pharmaceutical drug development include the combination of forward and reverse translation, the classification of disease conditions as multi-omics-defined endotypes, the integration of
AI- and algorithm-based R\&D concepts, the implementation of digital biomarkers as clinical endpoints and the development of companion diagnostics. The rise of datadriven and algorithm-based R\&D necessitates the establishment of a new mindset of how data mining and AI tools can be used effectively to discover and develop new drugs [111]. The near future will show whether and how these emerging AI-based digital tools will reveal new targets, pathogenic disease signatures, optimize clinical trial designs and overall impact drug development across pharmaceutical industries. Convergence of patient-centric real-world evidence (RWE) tools, EHRs, multi-omics profiling, digital biomarkers and AI-based data analysis will pave the way towards biomarker-enabled algorithmbased precision R\&D.

\section{Outlook}

The Translational Precision Medicine evolution comes with distinct challenges: (i) multi-omics data are mainly useful to drug discovery and development if they reveal new drug targets or biomarker signatures that correlate with disease outcome and/or treatment response [61]; (ii) multi-omics-based patient and disease stratification requires accurate diagnoses and detailed clinical annotations/EHRs; (iii) digital biomarkers as clinical endpoints provide objective and quantitative measures yet still require broader clinical use and health authority acceptance; (iv) biomarker-guided trial designs and precision medicine approaches are already widely implemented in oncology and rare diseases, while other non-oncology areas have just started to pursue these concepts and (v) precision medicine/companion diagnostics approaches come with substantial development costs and reimbursement hurdles. One important question is how these novel technologies and assessments are perceived by patients, as acceptance and adherence to clinical read-outs is key for patient trial recruitment and long-term engagement. Novel patient-centric interaction approaches are currently implemented to engage patients more pro-actively in R\&D, RWE networks and clinical trials. New cloudbased data systems and platforms for interactions with regulatory agencies [167], for sharing datasets between industry and academia, for public-private partnerships or for managing cross-industry partnerships and multidisciplinary initiatives like the Information Exchange and Data Transformation (INFORMED) initiative of the FDA [168] will further shape the way towards data-driven medicine.

The COVID-19 era substantially disrupted the traditional pharmaceutical R\&D approach at several layers [169-172]: (i) virtual, data-based, data-sharing (including open repositories such as bioRxiv and medRxiv) and collaborative research and drug discovery/ 
development concepts are getting higher traction; (ii) large longitudinal datasets collected from COVID19 patients are systematically analyzed and offer great potential for multi-omics [173-175] and AI-based analyses [169, 176-178], supporting diagnosis, basic disease understanding, endotyping, image analysis, drug target identification and drug repurposing [169, 179]; (iii) clinical trials are accelerated, decentralized and increasingly include digital endpoints, biosensors, home nursing, patient-centric sampling and remote clinical trial recruitment and monitoring strategies $[171,180]$, accompanied by a FDA guidance on conduct of clinical crials during COVID-19 [181]. In combination, these emerging concepts rapidly and successfully implemented during the COVID-19 outbreak hold promise to make drug discovery and development more efficient and less burdensome to patients also beyond the pandemic era.

Emerging therapeutic modalities, including CAR T-cells [182], gene therapy [183, 184], induced protein degradation [185] or mRNA-based principles [186, 187], and patient-derived organoids for ex vivo drug response testing to guide personalized treatments [188] add further levels of complexity to biomarkerguided translational precision. Finally, the core future challenge for Translational Precision Medicine as for drug development overall remains how to leverage and embrace new molecular and digital technologies in a way that is feasible for larger clinical trials, accepted by regulators and, most importantly, by patients.

\begin{abstract}
Abbreviations
Al: Artificial Intelligence; BEST: Biomarkers, Endpoints and other Tools; BQP: Biomarker qualification program; CLIA: Clinical Laboratory Improvement Amendments; CoU: Context-of-use; CTTI: Clinical Trials Transformation Initiative; EHR: Electronic health records; EMA: European Medicines Agency; FDA: U.S. Food and Drug Administration; IVD: In vitro diagnostic; LADME: Liberation, Absorption, Distribution, Metabolism, Excretion; MIDD: Model-Informed Drug Development; NIH: National Institutes of Health; PD: Pharmacodynamic; PHC: Personalised healthcare; PK: Pharmacokinetic; R\&D: Research and development; RWE: Real-world evidence; SOP: Standard operating procedures.
\end{abstract}

\section{Acknowledgements}

We thank all colleagues at Novartis and Roche for related discussions.

\section{Authors' contributions}

DH drafted, conceptualized and provided overall supervision for the manuscript, VdL, SC, EF, RS, MF, SA, LP contributed to specific sections and revised the manuscript, AK revised the manuscript, JL revised the manuscript. JM revised the manuscript, AS revised the manuscript, MH revised the manuscript. All authors read and approved the final manuscript.

\section{Funding}

Open Access funding enabled and organized by Projekt DEAL. No funding has been received for this publication.

\section{Availability of data and materials}

Not applicable.

\section{Declarations}

Ethics approval and consent to participate

Not applicable.

\section{Consent for publication}

Not applicable.

\section{Competing interests}

The authors declare that there are no competing interests. DH, VdL, AK, JL, SC, EF, RS, MF and MH work for Novartis, LP and AR work for Roche.

\section{Author details}

${ }^{1}$ Novartis Institutes for BioMedical Research, Basel, Switzerland. ${ }^{2}$ Department of Pediatrics I, University of Tübingen, Tübingen, Germany. ${ }^{3}$ Novartis Institutes for BioMedical Research, Cambridge, MA, USA. ${ }^{4}$ Basel, Switzerland. ${ }^{5}$ Novartis Precision Medicine, Cambridge, MA, USA. ${ }^{6}$ Reutlingen University, Reutlingen, Germany. ${ }^{7}$ Roche Innovation Center Basel, Basel, Switzerland.

Received: 2 February 2021 Accepted: 25 May 2021

Published online: 05 June 2021

\section{References}

1. Paul SM, Mytelka DS, Dunwiddie CT, Persinger CC, Munos BH, Lindborg SR, et al. How to improve R\&D productivity: the pharmaceutical industry's grand challenge. Nat Rev Drug Discov. 2010;9(3):203-14.

2. Cook D, Brown D, Alexander R, March R, Morgan P, Satterthwaite G, et al. Lessons learned from the fate of AstraZeneca's drug pipeline: a fivedimensional framework. Nat Rev Drug Discovery. 2014;13(6):419-31.

3. Dugger SA, Platt A, Goldstein DB. Drug development in the era of precision medicine. Nat Rev Drug Discov. 2018;17(3):183-96.

4. Seyhan AA, Carini C. Are innovation and new technologies in precision medicine paving a new era in patients centric care? J Transl Med. 2019;17:114.

5. Feldman AM. Bench-to-bedside; clinical and translational research; personalized medicine; precision medicine-what's in a name? Clin Transl Sci. 2015:8(3):171-3.

6. Hey SP, Gerlach CV, Dunlap G, Prasad V, Kesselheim AS. The evidence landscape in precision medicine. Sci Transl Med. 2020;12(540):eaaw7745

7. Zeggini $\mathrm{E}$, Gloyn AL, Barton AC, Wain LV. Translational genomics and precision medicine: moving from the lab to the clinic. Science. 2019;365(6460):1409-13.

8. Austin CP. Translating translation. Nat Rev Drug Discov. 2018;17(7):455-6.

9. Butler D. Translational research: crossing the valley of death. Nature. 2008;453(7197):840-2.

10. Tageja N. Bridging the translation gap - new hopes, new challenges. Fund Clin Pharmacol. 2011;25(2):163-71.

11. NIH. National Center for Advancing Translational Sciences (HCATS). https://ncats.nih.gov/.

12. Shahzad A, Don G, Cohrs RJ. Translational medicine definition by the European Society for Translational Medicine. New Horiz Transl Med. 2015;2(3):89-91.

13. Shakhnovich $\mathrm{V}$. It's time to reverse our thinking: the reverse translation research paradigm. Cts-Clin Transl Sci. 2018;11(2):98-9.

14. Shih HP, Zhang X, Aronov AM. Drug discovery effectiveness from the standpoint of therapeutic mechanisms and indications. Nat Rev Drug Discov. 2018;17(1):78.

15. Frank R, Hargreaves R. Clinical biomarkers in drug discovery and development. Nat Rev Drug Discov. 2003;2(7):566-80.

16. Kaur R, Chupp G. Phenotypes and endotypes of adult asthma: Moving toward precision medicine. J Allergy Clin Immun. 2019;144(1):1-12.

17. Dean K, Niven R. Asthma phenotypes and endotypes: implications for personalised therapy. BioDrugs. 2017;31(5):393-408.

18. Ricker CA, Crawford K, Matlock K, Lathara M, Seguin B, Rudzinski ER, et al. Defining an embryonal rhabdomyosarcoma endotype. Cold Spring Harb Mol Case Stud. 2020;6(2):a005066. 
19. Toward precision medicine: building a knowledge network for biomedical research and a new taxonomy of disease. The National Academies Collection: Reports funded by National Institutes of Health. Washington, DC; 2011. https://pubmed.ncbi.nlm.nih.gov/22536618/.

20. Shah $\mathrm{NH}$, Tenenbaum JD. The coming age of data-driven medicine: translational bioinformatics' next frontier. J Am Med Inform Assn. 2012;19(E1):E2-4.

21. Mayo CS, Deasy JO, Chera BS, Freymann J, Kirby JS, Hardenberg PH. How can we effect culture change toward data-driven medicine? Int J Radiat Oncol. 2016;95(3):916-21.

22. Sharma N, Cutting GR. The genetics and genomics of cystic fibrosis. J Cyst Fibros. 2020;19:S5-9.

23. Stratton MR, Campbell PJ, Futreal PA. The cancer genome. Nature. 2009:458(7239):719-24.

24. Torkamani A, Wineinger NE, Topol EJ. The personal and clinical utility of polygenic risk scores. Nat Rev Genet. 2018;19(9):581-90.

25. Li R, Chen Y, Ritchie MD, Moore JH. Electronic health records and polygenic risk scores for predicting disease risk. Nat Rev Genet. 2020;21(8):493-502.

26. Petricoin EF, Zoon KC, Kohn EC, Barrett JC, Liotta LA. Clinical proteomics: translating benchside promise into bedside reality. Nat Rev Drug Discov. 2002;1(9):683-95.

27. Dupree EJ, Jayathirtha M, Yorkey H, Mihasan M, Petre BA, Darie CC. A critical review of bottom-up proteomics: the good, the bad, and the future of this field. Proteomes. 2020;8(3):14.

28. Lamb JR, Jennings LL, Gudmundsdottir V, Gudnason V, Emilsson V. It's in our blood: a glimpse of personalized medicine. Trends Mol Med. 2020;27(1):20-30.

29. Gold L, Walker JJ, Wilcox SK, Williams S. Advances in human proteomics at high scale with the SOMAscan proteomics platform. N Biotechnol. 2012;29(5):543-9.

30. Jacob J, Ngo D, Finkel N, Pitts R, Gleim S, Benson MD, et al. Application of large-scale aptamer-based proteomic profiling to planned myocardial infarctions. Circulation. 2018;137(12):1270-7.

31. Gudmundsdottir V, Zaghlool SB, Emilsson V, Aspelund T, Ilkov M, Gudmundsson EF, et al. Circulating protein signatures and causal candidates for type 2 diabetes. Diabetes. 2020;69(8):1843-53.

32. Emilsson V, Ilkov M, Lamb JR, Finkel N, Gudmundsson EF, Pitts R, et al. Co-regulatory networks of human serum proteins link genetics to disease. Science. 2018;361(6404):769-73.

33. Emilsson V, Gudnason V, Jennings LL. Predicting health and life span with the deep plasma proteome. Nat Med. 2019;25(12):1815-6.

34. Sun YV, Hu YJ. Integrative analysis of multi-omics data for discovery and functional studies of complex human diseases. Adv Genet. 2016;93:147-90

35. Hasin Y, Seldin M, Lusis A. Multi-omics approaches to disease. Genome Biol. 2017;18:83

36. Barabasi AL, Gulbahce N, Loscalzo J. Network medicine: a networkbased approach to human disease. Nat Rev Genet. 2011;12(1):56-68.

37. Cowen L, Ideker T, Raphael BJ, Sharan R. Network propagation: a universal amplifier of genetic associations. Nat Rev Genet. 2017;18(9):551-62.

38. Schork NJ. Personalized medicine: time for one-person trials. Nature. 2015:520(7549):609-11.

39. Forst CV. Network genomics-a novel approach for the analysis of biological systems in the post-genomic era. Mol Biol Rep. 2002;29(3):265-80.

40. Goh WW, Lee YH, Chung M, Wong L. How advancement in biological network analysis methods empowers proteomics. Proteomics. 2012;12(4-5):550-63.

41. Zhao S, lyengar R. Systems pharmacology: network analysis to identify multiscale mechanisms of drug action. Annu Rev Pharmacol Toxicol. 2012;52:505-21.

42. Ruiz-Godoy L, Enriquez-Carcamo V, Suarez-Roa L, Lopez-Castro ML, Santamaria A, Orozco-Morales M, et al. Identification of specific preanalytical quality control markers in plasma and serum samples. Anal Methods-Uk. 2019;11(17):2259-71

43. Papiez A, Marczyk M, Polanska J, Polanski A. Batchl: Batch effect Identification in high-throughput screening data using a dynamic programming algorithm. Bioinformatics. 2019;35(11):1885-92.

44. Yamada R, Okada D, Wang J, BasakT, Koyama S. Interpretation of omics data analyses. J Hum Genet. 2020;66(1):93-102.
45. Elefsinioti A, Bellaire T, Wang A, Quast K, Seidel H, Braxenthaler M, et al Key factors for successful data integration in biomarker research. Nat Rev Drug Discov. 2016;15(6):369-70.

46. Hu YJ, An Q, Sheu K, Trejo B, Fan SX, Guo Y. Single cell multi-omics technology: methodology and application. Front Cell Dev Biol. 2018;6:93-102.

47. Verheijen M, Tong WD, Shi LM, Gant TW, Seligman B, Caiment F. Towards the development of an omics data analysis framework. Regul Toxicol Pharm. 2020;112:104621.

48. Kraus VB. Biomarkers as drug development tools: discovery, validation, qualification and use. Nat Rev Rheumatol. 2018;14(6):354-62.

49. Baker M. Biorepositories: Building better biobanks. Nature. 2012:486(7401):141-6.

50. Wilkinson MD, Dumontier M, Aalbersberg IJ, Appleton G, Axton M, Baak A, et al. The FAIR Guiding Principles for scientific data management and stewardship. Sci Data. 2016;3:160018.

51. BEST (Biomarkers, EndpointS, and other Tools) Resource. https://www. ncbi.nlm.nih.gov/books/NBK326791/.

52. Roberts SF, Fischhoff MA, Sakowski SA, Feldman EL. Perspective: transforming science into medicine: how clinician-scientists can build bridges across research's "Valley of Death." Acad Med. 2012;87(3):266-70.

53. Akhtar A. The flaws and human harms of animal experimentation. Camb Q Healthc Ethic. 2015;24(4):407-19.

54. Seyhan AA, Carini C. Are innovation and new technologies in precision medicine paving a new era in patients centric care? J Transl Med. 2019;17(1):114.

55. Lassere MN, Johnson KR, Boers $M$, Tugwell P, Brooks $P$, Simon $L$, et al. Definitions and validation criteria for biomarkers and surrogate endpoints: Development and testing of a quantitative hierarchical levels of evidence schema. J Rheumatol. 2007;34(3):607-15.

56. Bravo-Merodio L, Williams JA, Gkoutos GV, Acharjee A. Omics biomarker identification pipeline for translational medicine. J Transl Med. 2019;17:155.

57. FDA. https://www.fda.gov/drugs/development-approval-processdrugs/drug-development-tool-ddt-qualification-programs.

58. FDA. https://www.fda.gov/drugs/biomarker-qualification-program/listqualified-biomarkers.

59. Williams SA, Slavin DE, Wagner JA, Webster CJ. A cost-effectiveness approach to the qualification and acceptance of biomarkers. Nat Rev Drug Discov. 2006;5(11):897-902.

60. Trusheim MR, Berndt ER, Douglas FL. Stratified medicine: strategic and economic implications of combining drugs and clinical biomarkers. Nat Rev Drug Discov. 2007;6(4):287-93.

61. Hurko O, Jones GK. Valuation of biomarkers. Nat Rev Drug Discov. 2011;10(4):253-4.

62. Mandrekar SJ, Sargent DJ. Clinical trial designs for predictive biomarker validation: one size does not fit all. J Biopharm Stat. 2009;19(3):530-42.

63. Antoniou M, Kolamunnage-Dona R, Jorgensen AL. Biomarker-guided non-adaptive trial designs in phase II and phase III: a methodological review. J Pers Med. 2017;7(1):1.

64. Park JJH, Siden E, Zoratti MJ, Dron L, Harari O, Singer J, et al. Systematic review of basket trials, umbrella trials, and platform trials: a landscape analysis of master protocols. Trials. 2019;20(1):572.

65. http://www.bigted.org.

66. Ivison S, Des Rosiers C, Lesage S, Rioux JD, Levings MK. Biomarkerguided stratification of autoimmune patients for biologic therapy. Curr Opin Immunol. 2017:49:56-63.

67. Freidlin B, Korn EL. Biomarker enrichment strategies: matching trial design to biomarker credentials. Nat Rev Clin Oncol. 2014;11(2):81-90.

68. FDA. https://www.fda.gov/regulatory-information/search-fda-guida nce-documents/enrichment-strategies-clinical-trials-support-approvalhuman-drugs-and-biological-products.

69. Dickson D, Johnson J, Bergan R, Owens R, Subbiah V, Kurzrock R. The master observational trial: a new class of master protocol to advance precision medicine. Cell. 2020;180(1):9-14.

70. Kalaitzopoulos D. The potential of precision medicine. New Horiz Transl Med. 2016;3(2):63-5.

71. Mullard A. $\$ 215$ million precision-medicine initiative takes shape. Nat Rev Drug Disc. 2015;14(3):155.

72. Collins FS, Varmus H. A new initiative on precision medicine. New Engl J Med. 2015:372(9):793-5. 
73. Doble B, Tan M, Harris A, Lorgelly P. Modeling companion diagnostics in economic evaluations of targeted oncology therapies: systematic review and methodological checklist. Expert Rev Mol Diagn. 2015;15(2):235-54.

74. Jorgensen JT. Companion diagnostics: the key to personalized medicine. Expert Rev Mol Diagn. 2015;15(2):153-6.

75. Mankoff DA, Edmonds CE, Farwell MD, Pryma DA. Development of companion diagnostics. Semin Nucl Med. 2016;46(1):47-56.

76. Hersom M, Jorgensen JT. Companion and complementary diagnosticsfocus on PD-L1 expression assays for PD-1/PD-L1 checkpoint inhibitors in non-small cell lung cancer. Ther Drug Monit. 2018;40(1):9-16.

77. Lin E, Chien J, Ong FS, Fan JB. Challenges and opportunities for nextgeneration sequencing in companion diagnostics. Expert Rev Mol Diagn. 2015;15(2):193-209.

78. Tsourounis M, Stuart J, Pignato W, Toscani M, Barone J. Current trends in personalized medicine and companion diagnostics: a summary from the DIA meeting on personalized medicine and companion diagnostics. Ther Innov Regul Sci. 2015;49(4):530-43.

79. FDA. https://www.fda.gov/medical-devices/in-vitro-diagnostics/listcleared-or-approved-companion-diagnostic-devices-in-vitro-and-imagi ng-tools.

80. Mansfield EA. FDA perspective on companion diagnostics: an evolving paradigm. Clin Cancer Res. 2014;20(6):1453-7.

81. FDA. https://www.fda.gov/medical-devices/in-vitro-diagnostics/compa nion-diagnostics.

82. Milne CP, Bryan C, Garafalo S, McKiernan M. Complementary versus companion diagnostics: apples and oranges? Biomark Med. 2015;9(1):25-34.

83. Jorgensen JT. Companion and complementary diagnostics: clinical and regulatory perspectives. Trends Cancer. 2016;2(12):706-12.

84. Khan Z, Di Nucci F, Kwan A, Hammer C, Mariathasan S, Rouilly $V$, et al. Polygenic risk for skin autoimmunity impacts immune checkpoint blockade in bladder cancer. Proc Natl Acad Sci USA. 2020;117(22):12288-94.

85. Koido M, Kawakami E, Fukumura J, Noguchi Y, Ohori M, Nio Y, et al. Polygenic architecture informs potential vulnerability to drug-induced liver injury. Nat Med. 2020;26(10):1541-8.

86. Love-Koh J, Peel A, Rejon-Parrilla JC, Ennis K, Lovett R, Manca A, et al. The future of precision medicine: potential impacts for health technology assessment. Pharmacoeconomics. 2018;36(12):1439-51.

87. Filipp FV. Opportunities for artificial intelligence in advancing precision medicine. Curr Genet Med Rep. 2019;7(4):208-13.

88. Pettitt D, Smith J, Meadows N, Arshad Z, Schuh A, DiGiusto D, et al. Regulatory barriers to the advancement of precision medicine. Expert Rev Precis Me. 2016;1(3):319-29.

89. Vicini P, Gastonguay MR, Foster DM. Model-based approaches to biomarker discovery and evaluation: a multidisciplinary integrated review. Crit Rev Biomed Eng. 2002;30(4-6):379-418.

90. Paalzow LK. Torsten Teorell, the father of pharmacokinetics. Ups J Med Sci. 1995;100(1):41-6.

91. Sheiner LB, Stanski DR, Vozeh S, Miller RD, Ham J. Simultaneous modeling of pharmacokinetics and pharmacodynamics: application to d-tubocurarine. Clin Pharmacol Ther. 1979;25(3):358-71.

92. Holford NH. Clinical pharmacokinetics and pharmacodynamics of warfarin. Understanding the dose-effect relationship Clin Pharmacokinet. 1986;11(6):483-504.

93. Anderson BJ, Holford NH. Mechanism-based concepts of size and maturity in pharmacokinetics. Annu Rev Pharmacol Toxicol. 2008:48:303-32.

94. Darwich AS, Margolskee A, Pepin X, Aarons L, Galetin A, RostamiHodjegan A, et al. IMI - Oral biopharmaceutics tools project - Evaluation of bottom-up PBPK prediction success part 3: Identifying gaps in system parameters by analysing In Silico performance across different compound classes. Eur J Pharm Sci. 2017;96:626-42.

95. Krauss M, Mueller C, Schlender J, Schuppert A, Block M, Kuepfer L. A systems pharmacology approach for translational learning and pharmakokinetic predictions across patient populations. J Pharmacokinet Phar. 2016:43:S67-8.

96. FDA. https://www.fda.gov/drugs/development-resources/model-infor med-drug-development-pilot-program.
97. Workgroup EM, Marshall SF, Burghaus R, Cosson V, Cheung SY, Chenel $M$, et al. Good practices in model-informed drug discovery and development: practice, application, and documentation. CPT Pharmacomet Syst Pharmacol. 2016;5(3):93-122.

98. Wang Y, Zhu H, Madabushi R, Liu Q, Huang SM, Zineh I. Model-informed drug development: current US regulatory practice and future considerations. Clin Pharmacol Ther. 2019;105(4):899-911.

99. Mak KK, Pichika MR. Artificial intelligence in drug development: present status and future prospects. Drug Discov Today. 2019;24(3):773-80.

100. Ching T, Himmelstein DS, Beaulieu-Jones BK, Kalinin AA, Do BT, Way GP, et al. Opportunities and obstacles for deep learning in biology and medicine. J R Soc Interface. 2018;15(141):20170387.

101. Vamathevan J, Clark D, Czodrowski P, Dunham I, Ferran E, Lee G, et al. Applications of machine learning in drug discovery and development. Nat Rev Drug Discov. 2019;18(6):463-77.

102. Ferrero E, Dunham I, Sanseau P. In silico prediction of novel therapeutic targets using gene-disease association data. J Transl Med. 2017;15:1-6.

103. Rouillard AD, Hurle MR, Agarwal P. Systematic interrogation of diverse Omic data reveals interpretable, robust, and generalizable transcriptomic features of clinically successful therapeutic targets. Plos Comput Biol. 2018;14(5):e1006142.

104. Patel-Murray NL, Adam M, Huynh N, Wassie BT, Milani P, Fraenkel E. A multi-omics interpretable machine learning model reveals modes of action of small molecules. Sci Rep. 2020;10(1):954.

105. Swan AL, Mobasheri A, Allaway D, Liddell S, Bacardit J. Application of machine learning to proteomics data: classification and biomarker identification in postgenomics biology. OMICS. 2013;17(12):595-610.

106. Bravo-Merodio L, Williams JA, Gkoutos GV, Acharjee A. Omics biomarker identification pipeline for translational medicine. J Transl Med. 2019;17(1):155.

107. Sharifi-Noghabi H, Zolotareva O, Collins CC, Ester M. MOLI: multi-omics late integration with deep neural networks for drug response prediction. Bioinformatics. 2019;35(14):i501-9.

108. Exarchos KP, Beltsiou M, Votti CA, Kostikas K. Artificial intelligence techniques in asthma: a systematic review and critical appraisal of the existing literature. Eur Respir J. 2020;56(3):2000521.

109. Zhang H, Deng K, Li H, Albin RL, Guan Y. Deep learning identifies digital biomarkers for self-reported Parkinson's disease. Patterns. 2020;1(3):100042.

110. Guthrie NL, Carpenter J, Edwards KL, Appelbaum KJ, Dey S, Eisenberg $\mathrm{DM}$, et al. Emergence of digital biomarkers to predict and modify treatment efficacy: machine learning study. Bmj Open. 2019;9(7):e030710.

111. Schneider P, Walters WP, Plowright AT, Sieroka N, Listgarten J, Goodnow RA Jr, et al. Rethinking drug design in the artificial intelligence era. Nat Rev Drug Discov. 2020;19(5):353-64.

112. Schuhmacher A, Gatto A, Hinder M, Kuss M, Gassmann O. The upside of being a digital pharma player. Drug Discov Today. 2020;25(9):1569-74.

113. Bera K, Schalper KA, Rimm DL, Velcheti V, Madabhushi A. Artificial intelligence in digital pathology - new tools for diagnosis and precision oncology. Nat Rev Clin Oncol. 2019;16(11):703-15.

114. Huang S, Yang J, Fong S, Zhao Q. Artificial intelligence in cancer diagnosis and prognosis: opportunities and challenges. Cancer Lett. 2020:471:61-71.

115. Subramanian M, Wojtusciszyn A, Favre L, Boughorbel S, Shan J, Letaief $\mathrm{KB}$, et al. Precision medicine in the era of artificial intelligence: implications in chronic disease management. J Transl Med. 2020;18(1):472.

116. Benjamens S, Dhunnoo P, Mesko B. The state of artificial intelligencebased FDA-approved medical devices and algorithms: an online database. Npj Digit Med. 2020;3:118.

117. FDA. https://www.fda.gov/medical-devices/software-medical-devicesamd/artificial-intelligence-and-machine-learning-software-medic al-device.

118. Mamoshina P, Vieira A, Putin E, Zhavoronkov A. Applications of deep learning in biomedicine. Mol Pharm. 2016;13(5):1445-54.

119. Perry B, Herrington W, Goldsack JC, Grandinetti CA, Vasisht KP, Landray $M J$, et al. Use of mobile devices to measure outcomes in clinical research, 2010-2016: a systematic literature review. Digit Biomark. 2018;2(1):11-30.

120. Bakker JP, Goldsack JC, Clarke M, Coravos A, Geoghegan C, Godfrey A, et al. A systematic review of feasibility studies promoting the use of mobile technologies in clinical research. NPJ Digit Med. 2019;2(1):47. 
121. Marra C, Chen JL, Coravos A, Stern AD. Quantifying the use of connected digital products in clinical research. NPJ Digit Med. 2020;3(1):50.

122. Arnerić SP, Cedarbaum JM, Khozin S, Papapetropoulos S, Hill DL, Ropacki $\mathrm{M}$, et al. Biometric monitoring devices for assessing end points in clinical trials: developing an ecosystem. Nat Rev Drug Discov. 2017; 16(10):736

123. Coravos A, Khozin S, Mandl KD. Developing and adopting safe and effective digital biomarkers to improve patient outcomes. NPJ Digit Med. 2019:2(1):14

124. Dorsey ER. A digital journal for a digital era. Digit Biomark. 2017;1:1-3.

125. Sim I. Mobile devices and health. N Engl J Med. 2019;381(10):956-68.

126. Dodge HH, Zhu J, Mattek NC, Austin D, Kornfeld J, Kaye JA. Use of highfrequency in-home monitoring data may reduce sample sizes needed in clinical trials. PLoS ONE. 2015;10(9):e0138095.

127. Cohen AB, Mathews SC. The digital outcome measure. Digit Biomark. 2018;2(3):94-105

128. Khozin S, Coravos A. Decentralized trials in the age of real-world evidence and inclusivity in clinical investigations. Clin Pharmacol Therapeutics. 2019;106:25-7.

129. Rodarte C. Pharmaceutical perspective: how digital biomarkers and contextual data will enable therapeutic environments. Digit Biomark. 2017;1(1):73-81.

130. Dorsey ER, Papapetropoulos S, Xiong M, Kieburtz K. The First Frontier: Digital Biomarkers for Neurodegenerative Disorders. Digit Biomark. 2017;1(1):6-13.

131. Lipsmeier F, Taylor Kl, Kilchenmann T, Wolf D, Scotland A, SchjodtEriksen J, et al. Evaluation of smartphone-based testing to generate exploratory outcome measures in a phase 1 Parkinson's disease clinical trial. Mov Disord. 2018;33(8):1287-97.

132. Zhan A, Mohan S, Tarolli C, Schneider RB, Adams JL, Sharma S, et al. Using smartphones and machine learning to quantify Parkinson disease severity: the mobile Parkinson disease score. JAMA Neurol. 2018;75(7):876-80.

133. Sieberts SK, Schaff J, Duda M, Pataki BÁ, Sun M, Snyder P, et al. Crowdsourcing digital health measures to predict Parkinson's disease severity: the Parkinson's Disease Digital Biomarker DREAM Challenge. bioRxiv. 2020;4(1):53.

134. Ritchie K, Carriere I, Su L, O'Brien JT, Lovestone S, Wells K, et al. The midlife cognitive profiles of adults at high risk of late-onset Alzheimer's disease: The PREVENT study. Alzheimers Dement. 2017;13(10):1089-97.

135. Kourtis LC, Regele OB, Wright JM, Jones GB. Digital biomarkers for Alzheimer's disease: the mobile/wearable devices opportunity. NPJ Dig Med. 2019;2(1):9.

136. Andrzejewski KL, Dowling AV, Stamler D, Felong TJ, Harris DA, Wong C, et al. Wearable sensors in Huntington disease: a pilot study. J Hunting ton's Dis. 2016;5(2):199-206.

137. Goldsack JC, Coravos A, Bakker JP, Bent B, Dowling AV, Fitzer-Attas C, et al. Verification, analytical validation, and clinical validation (V3): the foundation of determining fit-for-purpose for Biometric Monitoring Technologies (BioMeTs). NPJ Digit Med. 2020;3(1):55.

138. CTTI. https://www.ctti-clinicaltrials.org/blog/category/mobile-clini cal-trials.

139. DiMe. https://playbook.dimesociety.org/.

140. Cerreta F, Ritzhaupt A, Metcalfe T, Askin S, Duarte J, Berntgen M, et al. Digital technologies for medicines: shaping a framework for success. Nat Rev Drug Discov. 2020;19:573-4.

141. Kruizinga MD, Stuurman FE, Exadaktylos V, Doll RJ, Stephenson DT, Groeneveld GJ, et al. Development of novel, value-based, digital endpoints for clinical trials: a structured approach toward fit-for-purpose validation. Pharmacol Rev. 2020;72(4):899-909.

142. DBDP. https://dbdp.org/.

143. Bionetworks S. https://sagebionetworks.org/challenges-benchmarki $\mathrm{ng} /$.

144. MOBILISE-D. https://www.mobilise-d.eu/.

145. IDEA-FAST. https://idea-fast.eu/.

146. RADAR-AD. https://www.radar-ad.org/

147. Insel TR. Digital phenotyping: technology for a new science of behavior. JAMA. 2017;318(13):1215-6

148. Huckvale K, Venkatesh S, Christensen H. Toward clinical digital phenotyping: a timely opportunity to consider purpose, quality, and safety. NPJ Digit Med. 2019;2(1):88.
149. Fagherazzi G. Deep digital phenotyping and digital twins for precision health: time to dig deeper. J Med Internet Res. 2020;22(3):e16770.

150. Geissler J, Ryll B, di Priolo SL, Uhlenhopp M. Improving patient involvement in medicines research and development: a practical roadmap. Ther Innov Regul Sci. 2017;51(5):612-9.

151. Haerry D, Landgraf C, Warner K, Hunter A, Klingmann I, May M, et al. EUPATI and patients in medicines research and development: guidance for patient involvement in regulatory processes. Front Med. 2018;5:230.

152. Mavris $M$, Furia Helms $A$, Bere N. Engaging patients in medicines regulation: a tale of two agencies. Nat Rev Drug Discov. 2019;18(12):885-6.

153. Crocker JC, Ricci-Cabello I, Parker A, Hirst JA, Chant A, Petit-Zeman S, et al. Impact of patient and public involvement on enrolment and retention in clinical trials: systematic review and meta-analysis. BMJ. 2018;363:k4738.

154. Hoos A, Anderson J, Boutin M, Dewulf L, Geissler J, Johnston G, et al. Partnering with patients in the development and lifecycle of medicines: a call for action. Ther Innov Regul Sci. 2015;49(6):929-39.

155. Getz K. Reflections on the evolution of patient engagement in drug development. Pharmaceut Med. 2019;33(3):179-85.

156. PCORI. https://www.pcori.org/sites/default/files/Engagement-Rubric. pdf.

157. Boutin M, Dewulf L, Hoos A, Geissler J, Todaro V, Schneider RF, et al. Culture and process change as a priority for patient engagement in medicines development. Ther Innov Regul Sci. 2017;51(1):29-38.

158. Chalmers I, Bracken MB, Djulbegovic B, Garattini S, Grant J, Gulmezoglu $A M$, et al. How to increase value and reduce waste when research priorities are set. Lancet. 2014;383(9912):156-65.

159. Achieving patient-led research. https://www.nature.com/collections/ jchaaeijid.

160. Maccarthy J, Guerin S, Wilson AG, Dorris ER. Facilitating public and patient involvement in basic and preclinical health research. PLOS ONE. 2019;14(5):e0216600.

161. Chawla NV, Davis DA. Bringing big data to personalized healthcare: a patient-centered framework. J Gen Intern Med. 2013;28(Suppl 3):S660-5.

162. CTTI. https://www.ctti-clinicaltrials.org/briefing-room/recom mendations/ctti-recommendations-effective-engagement-patie nt-groups-around.

163. Garg S, Williams NL, Ip A, Dicker AP. Clinical integration of digital solutions in health care: an overview of the current landscape of digital technologies in cancer care. JCO Clin Cancer Inform. 2018;2:1-9.

164. Dinh-Le C, Chuang R, Chokshi S, Mann D. Wearable health technology and electronic health record integration: scoping review and future directions. JMIR Mhealth Uhealth. 2019;7(9):e12861.

165. Communication from the Commission to the European Parliament, the Council, the European Economic and Social Committee and the Committee of the Regions; A European Strategy for Data. https://ec.europa. eu/info/sites/default/files/communication-european-strategy-data19feb2020_en.pdf.

166. Khoury MJ, Bowen MS, Clyne M, Dotson WD, Gwinn ML, Green RF, et al. From public health genomics to precision public health: a 20-year journey. Genet Med. 2018;20(6):574-82.

167. Robertson AS, Malone H, Bisordi F, Fitton H, Garner C, Holdsworth S, et al. Cloud-based data systems in drug regulation: an industry perspective. Nat Rev Drug Discov. 2020;19(6):365-6.

168. Khozin S, Pazdur R, Shah A. INFORMED: an incubator at the US FDA for driving innovations in data science and agile technology. Nat Rev Drug Discovery. 2018;17(8):529-30.

169. Zhou Y, Wang F, Tang J, Nussinov R, Cheng F. Artificial intelligence in COVID-19 drug repurposing. Lancet Digit Health. 2020;2(12):e667-76.

170. Ting DSW, Carin L, Dzau V, Wong TY. Digital technology and COVID-19. Nat Med. 2020;26(4):459-61.

171. Shi J, Xiao Y, Zhang Y, Geng D, Cong D, Shi KX, et al. Challenges of drug development during the COVID-19 pandemic: key considerations for clinical trial designs. Br J Clin Pharmacol. 2020;87(5):2170-85.

172. May M. 2021: research and medical trends in a post-pandemic world. Nat Med. 2020;26(12):1808-9.

173. Su Y, Chen D, Yuan D, Lausted C, Choi J, Dai CL, et al. Multi-Omics Resolves a Sharp Disease-State Shift between Mild and Moderate COVID-19. Cell. 2020;183(6):1479-95. 
174. Barh D, Tiwari S, Weener ME, Azevedo V, Goes-Neto A, Gromiha MM, et al. Multi-omics-based identification of SARS-CoV-2 infection biology and candidate drugs against COVID-19. Comput Biol Med. 2020;126:104051.

175. Shu T, Ning W, Wu D, Xu J, Han Q, Huang M, et al. Plasma proteomics identify biomarkers and pathogenesis of COVID-19. Immunity. 2020;53(5):1108-22.

176. Whetton AD, Preston GW, Abubeker S, Geifman N. Proteomics and informatics for understanding phases and identifying biomarkers in COVID-19 disease. J Proteome Res. 2020;19(11):4219-32.

177. Zhang K, Liu X, Shen J, Li Z, Sang Y, Wu X, et al. Clinically applicable Al system for accurate diagnosis, quantitative measurements, and prognosis of COVID-19 Pneumonia using computed tomography. Cell. 2020;181(6):1423-33.

178. Jin C, Chen W, Cao Y, Xu Z, Tan Z, Zhang X, et al. Development and evaluation of an artificial intelligence system for COVID-19 diagnosis. Nat Commun. 2020;11(1):5088.

179. Weisberg E, Parent A, Yang PL, Sattler M, Liu Q, Liu Q, et al. Repurposing of kinase inhibitors for treatment of COVID-19. Pharm Res. 2020;37(9):167.

180. Wong CK, Ho DTY, Tam AR, Zhou M, Lau YM, Tang MOY, et al. Artificial intelligence mobile health platform for early detection of COVID-19 in quarantine subjects using a wearable biosensor: protocol for a randomised controlled trial. Bmj Open. 2020;10(7):e038555.

181. FDA. https://www.fda.gov/regulatory-information/search-fda-guida nce-documents/fda-guidance-conduct-clinical-trials-medical-produ cts-during-covid-19-public-health-emergency.
182. Depil S, Duchateau P, Grupp SA, Mufti G, Poirot L. "Off-the-shelf" allogeneic CART cells: development and challenges. Nat Rev Drug Discov. 2020;19(3):185-99.

183. Cavazzana M, Bushman FD, Miccio A, Andre-Schmutz I, Six E. Gene therapy targeting haematopoietic stem cells for inherited diseases: progress and challenges. Nat Rev Drug Discov. 2019;18(6):447-62.

184. High KA, Roncarolo MG. Gene Therapy. N Engl J Med. 2019;381(5):455-64.

185. Lai AC, Crews CM. Induced protein degradation: an emerging drug discovery paradigm. Nat Rev Drug Discov. 2017;16(2):101-14.

186. Sahin U, Kariko K, Tureci O. mRNA-based therapeutics-developing a new class of drugs. Nat Rev Drug Discov. 2014;13(10):759-80.

187. Pardi N, Hogan MJ, Porter FW, Weissman D. mRNA vaccines - a new era in vaccinology. Nat Rev Drug Discov. 2018;17(4):261-79.

188. Berkers G, van Mourik P, Vonk AM, Kruisselbrink E, Dekkers JF, de Winterde Groot KM, et al. Rectal organoids enable personalized treatment of cystic fibrosis. Cell Rep. 2019;26(7):1701-8.

\section{Publisher's Note}

Springer Nature remains neutral with regard to jurisdictional claims in published maps and institutional affiliations.
Ready to submit your research? Choose BMC and benefit from:

- fast, convenient online submission

- thorough peer review by experienced researchers in your field

- rapid publication on acceptance

- support for research data, including large and complex data types

- gold Open Access which fosters wider collaboration and increased citations

- maximum visibility for your research: over $100 \mathrm{M}$ website views per year

At BMC, research is always in progress.

Learn more biomedcentral.com/submissions 